\title{
Research Article \\ Optimality Conditions of Vector Set-Valued Optimization Problem Involving Relative Interior
}

\author{
Zhiang Zhou' ${ }^{1,2}$ \\ ${ }^{1}$ College of Sciences, Shanghai University, Shanghai 200444, China \\ ${ }^{2}$ Department of Applied Mathematics, Chongqing University of Technology, Chongqing 400054, China
}

Correspondence should be addressed to Zhiang Zhou, zhi_ang@163.com

Received 26 October 2010; Revised 25 December 2010; Accepted 22 January 2011

Academic Editor: Sin E. Takahasi

Copyright (c) 2011 Zhiang Zhou. This is an open access article distributed under the Creative Commons Attribution License, which permits unrestricted use, distribution, and reproduction in any medium, provided the original work is properly cited.

Firstly, a generalized weak convexlike set-valued map involving the relative interior is introduced in separated locally convex spaces. Secondly, a separation property is established. Finally, some optimality conditions, including the generalized Kuhn-Tucker condition and scalarization theorem, are obtained.

\section{Introduction}

In mathematical programming, set-valued optimization is a very important topic. Since the 1980s, many authors have paid attention to it. Some international journals such as Set-Valued and Variational Analysis (original name: Set-Valued Analysis) were also established. Theories and applications are widely developed. Rong and $\mathrm{Wu}$ [1], Li [2], and Yang [3] and Yang [4] introduced cone convexlikeness, subconvexlikeness, generalized subconvexlikeness, and nearly subconvexlikeness, respectively. In these generalized convex set-valued maps, it is clear that nearly subconvexlikeness is the weakest. We find that, in the above-mentioned papers, the convex cone has a nonempty topological interior. However, it is possible that the topological interior of the convex cone is empty. For instance, if $C=\{(r, 0) \mid r \geq 0\} \subseteq R^{2}$, then the topological interior of $C$ is empty. In order to study some optimization problems which the convex cone has empty topological interior, we have to weaken the concept of the topological interior. Rockafellar [5] introduced the relative interior, which is the generalization of the topological interior. Based on the relative interior, Frenk and Kassay [6, 7] obtained Lagrangian duality theorems and Bot et al. [8] studied strong duality for generalized convex optimization problems. Borwein and Lewis [9] introduced the quasirelative interior. Bot et al. [10] studied the regularity conditions via quasi-relative interior in 
convex programming. However, we find that only a few papers $[11,12]$ are about set-valued optimization involving the relative interior. In this paper, we will further study set-valued optimization problems involving relative interior.

This paper is organized as follows. In Section 2, we give some preliminaries. In Section 3, a kind of generalized weak convexlike set-valued map involving relative interior is introduced, and a separation property is established. In Section 4, some optimality conditions, including the generalized Kuhn-Tucker condition and scalarization theorem, are obtained.

\section{Preliminaries}

Let $X, Y$, and $Z$ be three separated locally convex spaces, and let 0 denote the zero element for every space. Let $K$ be a nonempty subset of $Y$. The generated cone of $K$ is defined as cone $K=\{\lambda a \mid a \in K, \lambda \geq 0\}$. A cone $K \subseteq Y$ is said to be pointed if $K \cap(-K)=\{0\}$. A cone $K \subseteq Y$ is said to be nontrivial if $K \neq\{0\}$ and $K \neq Y$.

Let $Y^{*}$ and $Z^{*}$ stand for the topological dual space of $Y$ and $Z$, respectively. From now on, let $C$ and $D$ be nontrivial pointed closed-convex cones in $Y$ and $Z$, respectively. The topological dual cone $C^{+}$and strict topological dual cone $C^{+i}$ of $C$ are defined as

$$
\begin{gathered}
C^{+}=\left\{y^{*} \in Y^{*} \mid\left\langle y, y^{*}\right\rangle \geqslant 0, \forall y \in C\right\}, \\
C^{+i}=\left\{y^{*} \in Y^{*} \mid\left\langle y, y^{*}\right\rangle>0, \forall y \in C \backslash\{0\}\right\},
\end{gathered}
$$

where $\left\langle y, y^{*}\right\rangle$ denotes the value of the linear continuous functional $y^{*}$ at the point $y$. The meanings of $D^{+}$and $D^{+i}$ are similar.

Let $K$ be a nonempty subset of $Y$. We denote by $\mathrm{cl} K$, int $K$, and aff $K$ the closed hull, topological interior, and affine hull of $K$, respectively.

Definition 2.1 (see $[11,13]$ ). Let $K$ be a subset of $Y$. The relative interior of $K$ is the set

$\operatorname{ri} K=\{x \in K \mid$ there exists $U$, a neighborhood of $x$, such that $U \cap$ aff $K \subseteq K\}$.

Now, we give some basic properties about the relative interior.

Lemma 2.2. Let $K$ be a subset of $Y$. Let $k_{0} \in K, \bar{k} \in$ ri $K, \alpha \in R$, and $\lambda \in(0,1]$. Then,

(a) $\alpha \operatorname{ri} K=\operatorname{ri}(\alpha K)$;

(b) if $K$ is convex, then

$$
(1-\lambda) k_{0}+\lambda \bar{k} \in \operatorname{ri} K
$$

Proof. (a) Since $\alpha$ aff $K=\operatorname{aff}(\alpha K)$, it is clear that $\alpha \operatorname{ri} K=\operatorname{ri}(\alpha K)$;

(b) since $\bar{k} \in$ ri $K$, there exists $V$, a neighborhood of 0 , such that

$$
(\bar{k}+V) \cap \text { aff } K \subseteq K .
$$


By (2.4), we have

$$
(\lambda \bar{k}+\lambda V) \cap(\lambda \text { aff } K) \subseteq \lambda K
$$

It follows from (2.5) that

$$
\left((1-\lambda) k_{0}+\lambda \bar{k}+\lambda V\right) \cap\left((1-\lambda) k_{0}+\lambda \text { aff } K\right) \subseteq(1-\lambda) k_{0}+\lambda K
$$

It is clear that

$$
(1-\lambda) k_{0}+\lambda \text { aff } K=\operatorname{aff} K
$$

Since $K$ is convex, we have

$$
(1-\lambda) k_{0}+\lambda K \subseteq K
$$

By (2.6), (2.7), and (2.8), we obtain

$$
\left((1-\lambda) k_{0}+\lambda \bar{k}+\lambda V\right) \cap \text { aff } K \subseteq K
$$

which implies that

$$
(1-\lambda) k_{0}+\lambda \bar{k} \in \operatorname{ri} K
$$

Remark 2.3. By Lemma 2.2, if $K$ is a convex cone, then ri $K \cup\{0\}$ is a convex cone.

Lemma 2.4. If $K$ is a convex cone of $Y$, then

$$
K+\operatorname{ri} K \subseteq \operatorname{ri} K \text {. }
$$

Proof. If ri $K=\phi$, it is clear that the conclusion holds. If ri $K \neq \phi$, we have

$$
K+\operatorname{ri} K=2\left(\frac{1}{2} K+\frac{1}{2} \operatorname{ri} K\right) \subseteq 2 \operatorname{ri} K=\operatorname{ri} 2 K=\operatorname{ri} K
$$

where Lemma 2.2(b) is used in the first inclusion relation and Lemma 2.2(a) is used in the second equality.

Lemma 2.5 (see $[14,15])$. Let $W$ be a linear topological space and $w^{*}$ be a linear functional on $W$. $w^{*}$ is continuous if and only if $H=\left\{w \mid\left\langle w, w^{*}\right\rangle=0, w \in W\right\}$ is closed. If $H$ is not closed, $H$ is dense in $W$.

We will close this section by giving a separation theorem based on the relative interior. 
Lemma 2.6 (see [11]). Let $K \subseteq Y$ be a closed-convex set with $\mathrm{ri} K \neq \phi$. If $0 \notin$ ri $K$, then there exists $y^{*} \in Y^{*} \backslash\{0\}$ such that $\left\langle k, y^{*}\right\rangle \geq 0$ for each $k \in K$.

Remark 2.7. The following example will show that the closeness of $K$ cannot be deleted in Lemma 2.6.

Example 2.8. Let $Y$ be an infinite-dimensional normed space and $k^{*}$ be a non-continuous linear functional on $Y$. $K$ is defined as

$$
K=\left\{k \mid\left\langle k, k^{*}\right\rangle=1, k \in Y\right\}
$$

Since aff $K=K$, it is clear that $0 \notin$ ri $K=K$. By Lemma $2.5, K$ is not closed and $\mathrm{cl} K=Y$. Therefore, for any $y^{*} \in Y^{*} \backslash\{0\}, y^{*}$ cannot separate 0 and $K$.

Remark 2.9. Example 2.8 shows that, even if $K$ is a convex subset of $Y$, the expression that $\operatorname{ri}(\mathrm{cl} K)=$ ri $K$ does not hold generally.

\section{Separation Property}

From now on, we suppose that ri $C \neq \phi$ and ri $D \neq \phi$. Let $A$ be a nonempty subset of $X$ and $F: A \rightarrow 2^{Y}$ be a set-valued map on $A$. Write $F(A)=\cup_{x \in A} F(x)$.

Definition 3.1 (see [1]). Let $A$ be a nonempty subset of $X$. A set-valued map $F: A \rightarrow 2^{Y}$ is called $C$-convexlike on $A$ if the set $F(A)+C$ is convex.

In $[2,3,16,17]$, when $\operatorname{int} C \neq \phi, C$-subconvexlike map and generalized $C$ subconvexlike map were introduced, respectively. The following two definitions are generalizations of $C$-subconvexlike map and generalized $C$-subconvexlike map, respectively.

Definition 3.2 (see [12]). Let $A$ be a nonempty subset of $X$. A set-valued map $F: A \rightarrow 2^{Y}$ is called $C$-weak convexlike on $A$ if the set $F(A)+\operatorname{ri} C$ is convex.

Definition 3.3 (see [12]). Let $A$ be a nonempty subset of X. A set-valued map $F: A \rightarrow 2^{Y}$ is called generalized $C$-weak convexlike on $A$ if the set cone $F(A)+\operatorname{ri} C$ is convex.

Remark 3.4. By [12, Theorems 3.1 and 3.2], we have the following implications:

$C$-convexlikeness $\Rightarrow C$-weak convexlikeness $\Rightarrow$ generalized $C$-weak convexlikeness.

However, the following two examples show that the converse of the above implications is not generally true.

Example 3.5. Let $X=Y=R^{2}, C=\left\{\left(y_{1}, 0\right) \mid y_{1} \geq 0\right\}$, and $A=\{(1,0),(0,2)\}$. The set-valued map $F: A \rightarrow 2^{Y}$ is defined as follows:

$$
\begin{aligned}
& F(1,0)=\left\{\left(y_{1}, y_{2}\right) \mid 1<y_{1} \leq 2,0 \leq y_{2} \leq 1\right\} \cup\{(1,0),(1,1)\} \\
& F(0,2)=\left\{\left(y_{1}, y_{2}\right) \mid 1<y_{1} \leq 2,1 \leq y_{2} \leq 2\right\} \cup\{(1,2),(1,1)\} .
\end{aligned}
$$


It is clear that $F(A)+\operatorname{ri} C$ is convex and $F(A)+C$ is not convex. Therefore, $F$ is $C$-weak convexlike on $A$. However, $F$ is not $C$-convexlike on $A$.

Example 3.6. Let $X=Y=R^{2}, C=\left\{\left(y_{1}, 0\right) \mid y_{1} \geq 0\right\}$, and $A=\{(1,0),(0,2)\}$. The set-valued map $F: A \rightarrow 2^{Y}$ is defined as follows:

$$
\begin{aligned}
& F(1,0)=\left\{\left(y_{1}, y_{2}\right) \mid y_{1} \geq 0,1 \leq y_{2} \leq-y_{1}+2\right\} \\
& F(0,2)=\left\{\left(y_{1}, y_{2}\right) \mid y_{1} \geq 1,0 \leq y_{2} \leq-y_{1}+2\right\}
\end{aligned}
$$

It is clear that cone $F(A)+\operatorname{ri} C$ is convex and $F(A)+\operatorname{ri} C$ is not convex. Therefore, $F$ is generalized $C$-weak convexlike on $A$. However, $F$ is not $C$-weak convexlike on $A$.

Now, we consider the following two systems.

System 1: There exists $x_{0} \in A$ such that $F\left(x_{0}\right) \cap(-$ ri $C) \neq \phi$.

System 2: There exists $y^{*} \in C^{+} \backslash\{0\}$ such that $\left\langle y, y^{*}\right\rangle \geq 0$, for all $y \in F(A)$.

Theorem 3.7. Let $A$ be a nonempty subset of $X$.

(i) Suppose that $F: A \rightarrow 2^{Y}$ is generalized $C$-weak convexlike on $A$ and $\operatorname{ri}(\mathrm{cl}(\operatorname{cone} F(A)+$ $\operatorname{ri} C))=\operatorname{ri}(\operatorname{cone} F(A)+\operatorname{ri} C) \neq \phi$. If System 1 has no solution, then System 2 has solution.

(ii) If $y^{*} \in C^{+i}$ is a solution of System 2, then System 1 has no solution.

Proof. (i) Firstly, we assert that $0 \notin$ cone $F(A)+$ ri $C$. Otherwise, there exist $x_{0} \in A, \alpha \geq 0$ such that $0 \in \alpha F\left(x_{0}\right)+\operatorname{riC}$.

Case 1. If $\alpha=0$, then $0 \in \mathrm{ri} C$. Thus, there exists $U$, a neighborhood of 0 , such that

$$
U \cap \operatorname{aff} C \subseteq C
$$

Without loss of generality, we suppose that $U$ is symmetric. It follows from (3.3) that

$$
U \cap(-\operatorname{aff} C) \subseteq(-C)
$$

It is clear that aff $C$ is a linear subspace of $Y$. Therefore, aff $C=-\operatorname{aff} C$. By (3.4), we have

$$
U \cap \operatorname{aff} C \subseteq(-C)
$$

By (3.3) and (3.5), we obtain

$$
U \cap \operatorname{aff} C \subseteq C \cap(-C)
$$

Since $C$ is nontrivial, there exists $\bar{c} \in C \backslash\{0\}$. By the absorption of $U$, there exists $\lambda$, a sufficiently small positive number, such that

$$
\lambda \bar{c} \in U \cap \text { aff } C \subseteq C \cap(-C),
$$

which contradicts that $C$ is pointed. 
Case 2. If $\alpha>0$, there exists $y_{0} \in F\left(x_{0}\right)$ such that $-y_{0} \in(1 / \alpha)$ ri $C \subseteq$ ri $C$, which contradicts $F(x) \cap(-$ ri $C)=\phi$, for all $x \in A$.

Therefore, our assertion is true. Thus, we obtain

$$
0 \notin \operatorname{ri}(\mathrm{cl}(\operatorname{cone} F(A)+\operatorname{ri} C))
$$

Since $F$ is generalized $C$-weak convexlike on $A, \operatorname{cl}(\operatorname{cone} F(A)+\operatorname{ri} C)$ is a closed-convex set. By Lemma 2.6, there exists $y^{*} \in Y^{*} \backslash\{0\}$ such that

$$
\left\langle y, y^{*}\right\rangle \geq 0, \quad \forall y \in \operatorname{cl}(\text { cone } F(A)+\operatorname{ri} C)
$$

So,

$$
\left\langle\alpha F(x)+c, y^{*}\right\rangle \geq 0, \quad \forall x \in A, c \in \operatorname{riC}, \alpha \geq 0
$$

Letting $\alpha=0$ in (3.10), we obtain

$$
\left\langle c, y^{*}\right\rangle \geq 0, \quad \forall c \in \operatorname{ric}
$$

We assert that $y^{*} \in C^{+}$. Otherwise, there exists $c^{\prime} \in C$ such that $\left\langle c^{\prime}, y^{*}\right\rangle<0$, hence, $\left\langle\theta c^{\prime}, y^{*}\right\rangle<0$, for all $\theta>0$. By Lemma 2.4, we have

$$
\theta c^{\prime}+c \in \operatorname{riC}, \quad \forall c \in \operatorname{riC}
$$

It follows from (3.11) that

$$
\left\langle\theta c^{\prime}+c, y^{*}\right\rangle \geq 0, \quad \forall \theta>0, c \in \operatorname{ric} .
$$

Thus, we obtain

$$
\theta\left\langle c^{\prime}, y^{*}\right\rangle+\left\langle c, y^{*}\right\rangle \geq 0, \quad \forall \theta>0, c \in \operatorname{riC}
$$

On the other hand, (3.14) does not hold when $\theta>-\left\langle c, y^{*}\right\rangle /\left\langle c^{\prime}, y^{*}\right\rangle \geq 0$. Therefore, $\left\langle c, y^{*}\right\rangle \geq$ 0 , for all $c \in C$, that is, $y^{*} \in C^{+}$.

Letting $\alpha=1$ in (3.10), we have

$$
\left\langle F(x)+c, y^{*}\right\rangle \geq 0, \quad \forall x \in A, c \in \operatorname{riC} .
$$

Taking $c_{0} \in \operatorname{ri} C, \lambda_{n}>0, \lim _{n \rightarrow \infty} \lambda_{n}=0$, we have

$$
\left\langle F(x)+\lambda_{n} c_{0}, y^{*}\right\rangle \geq 0, \quad \forall x \in A, n \in N
$$

Limitting (3.16), we obtain $\left\langle F(x), y^{*}\right\rangle \geq 0$, for all $x \in A$. 
(ii) Since $y^{*} \in C^{+i}$ is a solution of System 2, we have

$$
\left\langle y, y^{*}\right\rangle \geq 0, \quad \forall y \in F(A)
$$

Now, we suppose that System 1 has solution. Then, there exists $x_{0} \in A$ such that $F\left(x_{0}\right) \cap$ $(-\operatorname{ri} C) \neq \phi$. Thus, there exists $y_{0} \in F\left(x_{0}\right)$ such that $-y_{0} \in \operatorname{riC}$. It is clear that $-y_{0} \neq 0$. So, we have

$$
\left\langle y_{0}, y^{*}\right\rangle<0
$$

which contradicts (3.17).

Remark 3.8. If $Y=R^{n}$, by [5, Theorems 6.2 and 6.3], the condition that $\operatorname{ri}(\operatorname{cl}(\operatorname{cone} F(A)+$ $\operatorname{ri} C))=\operatorname{ri}(\operatorname{cone} F(A)+\operatorname{ri} C) \neq \phi$ holds automatically. However, by Remark 2.9, it is possible that, the condition that $\operatorname{ri}(\mathrm{cl}(\operatorname{cone} F(A)+\operatorname{ri} C))=\operatorname{ri}(\operatorname{cone} F(A)+\operatorname{ri} C) \neq \phi$ does not hold. Therefore, our assumption is reasonable.

\section{Optimality Conditions}

Let $F: A \rightarrow 2^{Y}$ and $G: A \rightarrow 2^{Z}$ be two set-valued maps from $A$ to $Y$ and $Z$, respectively. Now, we consider the following vector optimization problem of set-valued maps:

$$
\begin{array}{ll}
\min & F(x) \\
\text { s.t. } & -G(x) \cap D \neq \phi .
\end{array}
$$

The feasible set of (VP) is defined by

$$
S=\{x \in A \mid-G(x) \cap D \neq \phi\} .
$$

Now, we define

$$
\begin{gathered}
W \operatorname{Min}(F(S), C)=\left\{y_{0} \in F(S) \mid y_{0}-y \notin \operatorname{riC}, \forall y \in F(S)\right\}, \\
P \operatorname{Min}(F(S), C)=\left\{y_{0} \in F(S) \mid(-C) \cap \operatorname{cl}\left(\operatorname{cone}\left(F(S)+C-y_{0}\right)\right)=\{0\}\right\} .
\end{gathered}
$$

Definition 4.1. A point $x_{0}$ is called a weakly efficient solution of (VP) if $x_{0} \in S$ and $F\left(x_{0}\right) \cap$ $W \operatorname{Min}(F(S), C) \neq \phi$. A point pair $\left(x_{0}, y_{0}\right)$ is called a weak minimizer of $(\mathrm{VP})$ if $y_{0} \in F\left(x_{0}\right) \cap$ $W \operatorname{Min}(F(S), C)$.

Definition 4.2. A point $x_{0}$ is called a Benson properly efficient solution of (VP) if $x_{0} \in S$ and $F\left(x_{0}\right) \cap P \operatorname{Min}(F(S), C) \neq \phi$. A point pair $\left(x_{0}, y_{0}\right)$ is called a Benson proper minimizer of (VP) if $y_{0} \in F\left(x_{0}\right) \cap P \operatorname{Min}(F(S), C)$.

Let $I(x)=F(x) \times G(x)$, for all $x \in A$. It is clear that $I$ is a set-valued map from $A$ to $Y \times Z$, where $Y \times Z$ is a seperated local convex space with nontrivial pointed closed-convex 
cone $C \times D$. The topological dual space of $Y \times Z$ is $Y^{*} \times Z^{*}$, and the topological dual cone of $C \times D$ is $C^{+} \times D^{+}$.

By Definition 3.3, we say that the set-valued map $I: A \rightarrow 2^{Y \times Z}$ is generalized $C \times D$ weak convexlike on $A$ if cone $I(A)+\operatorname{ri}(C \times D)$ is a convex set of $Y \times Z$.

Theorem 4.3. Let $\operatorname{ri}\left(\operatorname{cl}\left(\operatorname{cone}^{*}(A)+\operatorname{ri}(C \times D)\right)\right)=\operatorname{ri}\left(\operatorname{cone} I^{*}(A)+\operatorname{ri}(C \times D)\right) \neq \phi$. Suppose that the following conditions hold:

(i) $\left(x_{0}, y_{0}\right)$ is a weak minimizer of $(\mathrm{VP})$;

(ii) $I^{*}(x)$ is generalized $C \times D$-weak convexlike on $A$, where $I^{*}(x)=\left(F(x)-y_{0}\right) \times G(x)$.

Then, there exists $\left(y^{*}, z^{*}\right) \in C^{+} \times D^{+}$with $\left(y^{*}, z^{*}\right) \neq(0,0)$ such that

$$
\begin{gathered}
\inf _{x \in A}\left(\left\langle F(x), y^{*}\right\rangle+\left\langle G(x), z^{*}\right\rangle\right)=\left\langle y_{0}, y^{*}\right\rangle \\
\inf \left\langle G\left(x_{0}\right), z^{*}\right\rangle=0
\end{gathered}
$$

Proof. According to Definition 4.1, we have

$$
\left(y_{0}-F(S)\right) \cap \operatorname{ri} C=\phi
$$

It is clear that $I^{*}(x)=I(x)-\left(y_{0}, 0\right)$, for all $x \in A$. We assert that

$$
-I^{*}(x) \cap \operatorname{ri}(C \times D)=\phi, \quad \forall x \in A .
$$

Otherwise, there exists $\bar{x} \in A$ such that

$$
-I^{*}(\bar{x}) \cap \operatorname{ri}(C \times D) \neq \phi
$$

It is easy to check that $\operatorname{ri}(C \times D)=\operatorname{ri} C \times \operatorname{ri} D$. Therefore,

$$
-I^{*}(\bar{x}) \cap(\operatorname{riC} \times \operatorname{ri} D) \neq \phi .
$$

By (4.7), we obtain

$$
\begin{gathered}
\left(y_{0}-F(\bar{x})\right) \cap \operatorname{ri} C \neq \phi, \\
-G(\bar{x}) \cap \operatorname{ri} D \neq \phi .
\end{gathered}
$$

It follows from (4.9) that $\bar{x} \in S$. Thus, by (4.8), we have

$$
\left(y_{0}-F(S)\right) \cap \operatorname{riC} \neq \phi,
$$

which contradicts (4.4). Therefore, (4.5) holds. 
Journal of Inequalities and Applications

By Theorem 3.7, there exists $\left(y^{*}, z^{*}\right) \in C^{+} \times D^{+}$with $\left(y^{*}, z^{*}\right) \neq(0,0)$ such that

$$
\left\langle I^{*}(x),\left(y^{*}, z^{*}\right)\right\rangle \geq 0, \quad \forall x \in A .
$$

That is,

$$
\left\langle F(x), y^{*}\right\rangle+\left\langle G(x), z^{*}\right\rangle \geq\left\langle y_{0}, y^{*}\right\rangle, \quad \forall x \in A .
$$

Since $x_{0} \in S$, there exists $p \in G\left(x_{0}\right)$ such that $-p \in D$. Because $z^{*} \in D^{+}$, we obtain $\left\langle p, z^{*}\right\rangle \leq 0$. On the other hand, taking $x=x_{0}$ in (4.12), we get

$$
\left\langle y_{0}, y^{*}\right\rangle+\left\langle p, z^{*}\right\rangle \geq\left\langle y_{0}, y^{*}\right\rangle
$$

It follows that $\left\langle p, z^{*}\right\rangle \geq 0$. So, $\left\langle p, z^{*}\right\rangle=0$. Thus, we have

$$
\left\langle y_{0}, y^{*}\right\rangle \in\left\langle F\left(x_{0}\right), y^{*}\right\rangle+\left\langle G\left(x_{0}\right), z^{*}\right\rangle \text {. }
$$

Therefore, it follows from (4.12) and (4.14) that

$$
\inf _{x \in A}\left(\left\langle F(x), y^{*}\right\rangle+\left\langle G(x), z^{*}\right\rangle\right)=\left\langle y_{0}, y^{*}\right\rangle
$$

Finally, taking again $x=x_{0}$ in (4.12), we obtain

$$
\left\langle y_{0}, y^{*}\right\rangle+\left\langle G\left(x_{0}\right), z^{*}\right\rangle \geq\left\langle y_{0}, y^{*}\right\rangle
$$

So, $\left\langle G\left(x_{0}\right), z^{*}\right\rangle \geq 0$. We have shown that there exists $p \in G\left(x_{0}\right)$ such that $\left\langle p, z^{*}\right\rangle=0$. Thus, we have

$$
\inf \left\langle G\left(x_{0}\right), z^{*}\right\rangle=0
$$

The following example will be used to illustrate Theorem 4.3.

Example 4.4. Let $X=Y=Z=R^{2}, C=D=\left\{\left(y_{1}, 0\right) \mid y_{1} \geq 0\right\}$, and $A=\{(1,0),(1,2)\}$. The set-valued map $F: A \rightarrow 2^{Y}$ is defined as follows:

$$
\begin{gathered}
F(1,0)=\left\{\left(y_{1}, y_{2}\right) \mid y_{1}=1,0 \leq y_{2} \leq 1\right\}, \\
F(1,2)=\left\{\left(y_{1}, y_{2}\right) \mid y_{1}>1,0 \leq y_{2} \leq-y_{1}+2\right\} .
\end{gathered}
$$

The set-valued map $G: A \rightarrow 2^{Y}$ is defined as follows:

$$
\begin{gathered}
G(1,0)=\left\{\left(y_{1}, y_{2}\right) \mid y_{1} \leq 0,0 \leq y_{2} \leq y_{1}+1\right\} \\
G(1,2)=\left\{\left(y_{1}, y_{2}\right) \mid y_{1} \geq-1, y_{1}+1 \leq y_{2} \leq 1\right\} .
\end{gathered}
$$


Let $x_{0}=(1,0)$ and $y_{0}=(1,0) \in F\left(x_{0}\right)$. It is clear that all conditions of Theorem 4.3 are satisfied. Therefore, there exist $y^{*}:\left\langle\left(y_{1}, y_{2}\right), y^{*}\right\rangle=y_{1}+y_{2}$ and $z^{*}:\left\langle\left(y_{1}, y_{2}\right), z^{*}\right\rangle=-y_{1}+y_{2}$ such that

$$
\begin{gathered}
\inf _{x \in A}\left(\left\langle F(x), y^{*}\right\rangle+\left\langle G(x), z^{*}\right\rangle\right)=\left\langle y_{0}, y^{*}\right\rangle, \\
\inf \left\langle G\left(x_{0}\right), z^{*}\right\rangle=0 .
\end{gathered}
$$

Remark 4.5. Theorem 4.3 generalizes Theorem 3.1 of [2] and Theorem 4.2 of [3].

Theorem 4.6. Suppose that the following conditions hold:

(i) $x_{0} \in S$;

(ii) there exist $y_{0} \in F\left(x_{0}\right)$ and $\left(y^{*}, z^{*}\right) \in C^{+i} \times D^{+}$such that

$$
\inf _{x \in A}\left(\left\langle F(x), y^{*}\right\rangle+\left\langle G(x), z^{*}\right\rangle\right) \geq\left\langle y_{0}, y^{*}\right\rangle
$$

Then, $x_{0}$ is a weakly efficient solution of (VP).

Proof. By condition (ii), we have

$$
\left\langle F(x)-y_{0}, y^{*}\right\rangle+\left\langle G(x), z^{*}\right\rangle \geq 0, \quad \forall x \in A .
$$

Suppose to the contrary that $x_{0}$ is not a weakly efficient solution of (VP). Then, there exists $x^{\prime} \in S$ such that $\left(y_{0}-F\left(x^{\prime}\right)\right) \cap \operatorname{ri} C \neq \phi$. Therefore, there exists $t \in F\left(x^{\prime}\right)$ such that $y_{0}-t \in \operatorname{ri} C \subseteq$ $C \backslash\{0\}$. Thus, we obtain

$$
\left\langle t-y_{0}, y^{*}\right\rangle<0
$$

Since $x^{\prime} \in S$, there exists $q \in G\left(x^{\prime}\right)$ such that $-q \in D$. Hence,

$$
\left\langle q, z^{*}\right\rangle \leq 0
$$

Adding (4.23) to (4.24), we have

$$
\left\langle t-y_{0}, y^{*}\right\rangle+\left\langle q, z^{*}\right\rangle<0
$$

which contradicts (4.22). Therefore, $x_{0}$ is a weakly efficient solution of (VP).

The following example will be used to illustrate Theorem 4.6.

Example 4.7. Let $X=Y=Z=R^{2}, C=D=\left\{\left(y_{1}, 0\right) \mid y_{1} \geq 0\right\}$, and $A=\{(1,0),(1,2)\}$. The set-valued map $F: A \rightarrow 2^{Y}$ is defined as follows:

$$
\begin{aligned}
& F(1,0)=\left\{\left(y_{1}, y_{2}\right) \mid y_{1} \geq 1, y_{1} \leq y_{2} \leq 2\right\}, \\
& F(1,2)=\left\{\left(y_{1}, y_{2}\right) \mid y_{1} \leq 2,1 \leq y_{2} \leq y_{1}\right\} .
\end{aligned}
$$


The set-valued map $G: A \rightarrow 2^{Y}$ is defined as follows:

$$
\begin{gathered}
G(1,0)=\left\{\left(y_{1}, y_{2}\right) \mid-1 \leq y_{1} \leq 0, y_{2}=0\right\}, \\
G(1,2)=\left\{\left(y_{1}, y_{2}\right) \mid-1 \leq y_{1} \leq 0,0 \leq y_{2} \leq 1\right\} .
\end{gathered}
$$

Let $x_{0}=(1,0), y_{0}=(1,1) \in F\left(x_{0}\right),\left\langle\left(y_{1}, y_{2}\right), y^{*}\right\rangle=y_{1}+y_{2}$, and $\left\langle\left(y_{1}, y_{2}\right), z^{*}\right\rangle=-y_{1}$. It is clear that all conditions of Theorem 4.6 are satisfied. Therefore, $(1,0)$ is a weakly efficient solution of (VP).

Remark 4.8. Theorem 4.6 generalizes [2, Theorem 3.3].

Now, we consider the following scalar optimization problem $(\mathrm{VP})_{\varphi}$ of $(\mathrm{VP})$ :

$$
\begin{aligned}
\min & \langle F(x), \varphi\rangle \\
\text { s.t. } & x \in S,
\end{aligned}
$$

where $\varphi \in Y^{*} \backslash\{0\}$.

Definition 4.9. If $x_{0} \in S, y_{0} \in F\left(x_{0}\right)$ and

$$
\left\langle y_{0}, \varphi\right\rangle \leq\langle y, \varphi\rangle, \quad \forall y \in F(S)
$$

then $x_{0}$ and $\left(x_{0}, y_{0}\right)$ are called a minimal solution and a minimizer of $(\mathrm{VP})_{\varphi}$, respectively.

Lemma 4.10 (see [18]). Let $U_{1}, U_{2} \subset Y$ be two closed-convex cones such that $U_{1} \cap U_{2}=\{0\}$. If $U_{2}$ is pointed and locally compact, then $\left(-U_{1}^{+}\right) \cap U_{2}^{+i} \neq \phi$.

Lemma 4.11. If $V$ is a subset of $Y$, then

$$
\begin{aligned}
\text { (i) } \mathrm{cl}(\operatorname{cone}(V+\operatorname{ri} C)) & =\mathrm{cl}(\operatorname{cone} V+\operatorname{ri} C) \text {, } \\
\text { (ii) } \mathrm{cl}(\operatorname{cone}(V+\operatorname{ri} C)) & =\mathrm{cl}(\operatorname{cone}(V+C)) \text {. }
\end{aligned}
$$

Proof. (i) If $V=\phi$, it is obvious that

$$
\operatorname{cl}(\operatorname{cone}(V+\operatorname{ri} C))=\operatorname{cl}(\text { cone } V+\operatorname{ri} C) .
$$

If $V \neq \phi$, there exists $c \in \operatorname{ri} C$. It is clear that

$$
\lambda c \in \text { cone } V+\operatorname{ri} C, \quad \forall \lambda \in(0,+\infty) \text {. }
$$

Letting $\curlywedge \rightarrow 0$ in (4.30), we have

$$
0 \in \operatorname{cl}(\text { cone } V+\operatorname{ri} C) \text {. }
$$


Now, we will show that

$$
\operatorname{cone}(V+\operatorname{ri} C) \subseteq(\text { cone } V+\operatorname{ri} C) \cup\{0\}
$$

Let $y \in \operatorname{cone}(V+\operatorname{ri} C)$.

Case 1. If $y=0$, then $y \in($ cone $V+\operatorname{ric}) \cup\{0\}$.

Case 2. If $y \neq 0$, there exist $\alpha>0, v \in V$, and $\bar{c} \in \operatorname{ri} C$ such that

$$
y=\alpha(v+\bar{c})=\alpha v+\alpha \bar{c} \in \text { cone } V+\operatorname{ri} C \subseteq(\text { cone } V+\operatorname{ri} C) \cup\{0\} .
$$

Therefore, (4.32) holds. Since $Y$ is separated, by (4.31) and (4.32), we obtain

$$
\begin{aligned}
\operatorname{cl}(\text { cone }(V+\operatorname{ri} C)) & \subseteq \operatorname{cl}((\text { cone } V+\operatorname{ri} C) \cup\{0\}) \\
& =\operatorname{cl}(\text { cone } V+\operatorname{ri} C) \cup \operatorname{cl}\{0\} \\
& =\operatorname{cl}(\text { cone } V+\operatorname{ri} C) \cup\{0\} \\
& =\operatorname{cl}(\text { cone } V+\operatorname{riC}) .
\end{aligned}
$$

That is,

$$
\operatorname{cl}(\operatorname{cone}(V+\operatorname{ri} C)) \subseteq \operatorname{cl}(\text { cone } V+\operatorname{ric}) .
$$

Using the technique of Lemma 2.1 in [19], we easily obtain

$$
\text { cone } V+\operatorname{ri} C \subseteq \mathrm{cl}(\operatorname{cone}(V+\operatorname{ri} C)) \text {. }
$$

So,

$$
\operatorname{cl}(\text { cone } V+\operatorname{ri} C) \subseteq \operatorname{cl}(\operatorname{cone}(V+\operatorname{ri} C)) .
$$

By (4.35) and (4.37), we have

$$
\operatorname{cl}(\operatorname{cone}(V+\operatorname{ri} C))=\operatorname{cl}(\text { cone } V+\operatorname{ri} C) .
$$

(ii) It is obvious that

$$
\operatorname{cl}(\operatorname{cone}(V+\operatorname{ri} C)) \subseteq \operatorname{cl}(\operatorname{cone}(V+C))
$$

We will show that

$$
\text { cone }(V+C) \subseteq \mathrm{cl}(\text { cone }(V+\operatorname{ri} C)) \text {. }
$$


It is clear that (4.40) holds if $V=\phi$. Now, we suppose that $V \neq \phi$. Let $y \in \operatorname{cone}(V+C)$, then there exist $\lambda \geq 0, v \in V$, and $c \in C$ such that

$$
y=\lambda(v+c)
$$

Since ri $C \neq \phi$, there exists $c_{0} \in$ ri $C$. It follows from Lemma 2.4 that

$$
\frac{\lambda}{\alpha} c_{0}+y=\lambda\left(\frac{1}{\alpha} c_{0}+c+v\right) \in \operatorname{cone}(V+\operatorname{ric}), \quad \forall \alpha>0
$$

Letting $\alpha \rightarrow+\infty$ in (4.42), we have

$$
y \in \operatorname{cl}(\operatorname{cone}(V+\operatorname{ri} C))
$$

which implies that (4.40) holds. By (4.40), we obtain

$$
\operatorname{cl}(\operatorname{cone}(V+C)) \subseteq \operatorname{cl}(\text { cone }(V+\operatorname{ri} C))
$$

By (4.39) and (4.44), we have

$$
\operatorname{cl}(\operatorname{cone}(V+\operatorname{ri} C))=\operatorname{cl}(\operatorname{cone}(V+C))
$$

Theorem 4.12. Suppose that the following conditions hold:

(i) $C \subseteq Y$ is locally compact;

(ii) $\left(x_{0}, y_{0}\right)$ is a Benson proper minimizer of (VP);

(iii) $F-y_{0}$ is generalized $C$-weak convexlike on $S$.

Then, there exists $\varphi \in C^{+i}$ such that $\left(x_{0}, y_{0}\right)$ is a minimizer of $(V P)_{\varphi}$.

Proof. By condition (ii), we have

$$
(-C) \cap \operatorname{cl}\left(\operatorname{cone}\left(F(S)+C-y_{0}\right)\right)=\{0\}
$$

By Lemma 4.11 and condition (iii), we obtain that $\mathrm{cl}\left(\operatorname{cone}\left(F(S)+C-y_{0}\right)\right)$ is a closed-convex cone. Thus, conditions of Lemma 4.10 are satisfied. Therefore, there exists $\varphi \in C^{+i}$ such that

$$
\varphi \in\left(\operatorname{cl}\left(\operatorname{cone}\left(F(S)+C-y_{0}\right)\right)\right)^{+} .
$$

Since $F(S)-y_{0} \subseteq \operatorname{cl}\left(\operatorname{cone}\left(F(S)+C-y_{0}\right)\right)$, we obtain

$$
\left\langle y-y_{0}, \varphi\right\rangle \geq 0, \quad \forall y \in F(S)
$$


That is,

$$
\langle y, \varphi\rangle \geq\left\langle y_{0}, \varphi\right\rangle, \quad \forall y \in F(S)
$$

So, $\left(x_{0}, y_{0}\right)$ is a minimizer of $(\mathrm{VP})_{\varphi}$.

The following example will be used to illustrate Theorem 4.12 .

Example 4.13. Let $X=Y=Z=R^{2}, C=D=\left\{\left(y_{1}, 0\right) \mid y_{1} \geq 0\right\}$, and $A=\{(1,0),(1,2)\}$. The set-valued map $F: A \rightarrow 2^{Y}$ is defined as follows:

$$
\begin{gathered}
F(1,0)=\left\{\left(y_{1}, y_{2}\right) \mid y_{1} \geq 1,2 \leq y_{2} \leq-y_{1}+4\right\} \cup\{(1,1)\}, \\
F(1,2)=\left\{\left(y_{1}, y_{2}\right) \mid y_{1} \geq 2,1 \leq y_{2} \leq-y_{1}+4\right\} .
\end{gathered}
$$

The set-valued map $G: A \rightarrow 2^{Z}$ is defined as follows:

$$
\begin{aligned}
G(1,0) & =\left\{\left(y_{1}, y_{2}\right) \mid y_{1} \leq 0,0 \leq y_{2} \leq y_{1}+1\right\} \\
G(1,2) & =\left\{\left(y_{1}, y_{2}\right) \mid y_{1} \geq-1, y_{1}+1 \leq y_{2} \leq 1\right\}
\end{aligned}
$$

Let $x_{0}=(1,0), y_{0}=(1,1) \in F\left(x_{0}\right)$. Thus, all conditions of Theorem 4.12 are satisfied. Therefore, there exists $\varphi:\left\langle\left(y_{1}, y_{2}\right), \varphi\right\rangle=y_{1}+y_{2}$ such that $\left(x_{0}, y_{0}\right)$ is a minimizer of $(\mathrm{VP})_{\varphi}$.

Remark 4.14. Theorem 4.12 generalizes Theorem 4.2 of [16] and the necessity of Theorem 4.1 of [17].

In this paper, our results improve some results in the literature, and our results are very useful to form Lagrange multipliers rule and establish duality theory.

\section{Acknowledgments}

This paper was supported by the National Nature Science Foundation of China (Grant 10831009). The author would like to express his thanks to his supervisor Professor X. M. Yang for guidance and the referees for valuable comments and suggestions.

\section{References}

[1] W. D. Rong and Y. N. Wu, "Characterizations of super efficiency in cone-convexlike vector optimization with set-valued maps," Mathematical Methods of Operations Research, vol. 48, no. 2, pp. 247-258, 1998.

[2] Z. Li, "A theorem of the alternative and its application to the optimization of set-valued maps," Journal of Optimization Theory and Applications, vol. 100, no. 2, pp. 365-375, 1999.

[3] X. M. Yang, X. Q. Yang, and G. Y. Chen, "Theorems of the alternative and optimization with set-valued maps," Journal of Optimization Theory and Applications, vol. 107, no. 3, pp. 627-640, 2000.

[4] X. M. Yang, D. Li, and S. Y. Wang, "Near-subconvexlikeness in vector optimization with set-valued functions," Journal of Optimization Theory and Applications, vol. 110, no. 2, pp. 413-427, 2001.

[5] R. T. Rockafellar, Convex Analysis, Princeton Mathematical Series, No. 28, Princeton University Press, Princeton, NJ, USA, 1970. 
[6] J. B. G. Frenk and G. Kassay, "On classes of generalized convex functions, Gordan-Farkas type theorems, and Lagrangian duality," Journal of Optimization Theory and Applications, vol. 102, no. 2, pp. 315-343, 1999.

[7] J. B. G. Frenk and G. Kassay, "Lagrangian duality and cone convexlike functions," Journal of Optimization Theory and Applications, vol. 134, no. 2, pp. 207-222, 2007.

[8] R. I. Boţ, G. Kassay, and G. Wanka, "Strong duality for generalized convex optimization problems," Journal of Optimization Theory and Applications, vol. 127, no. 1, pp. 45-70, 2005.

[9] J. M. Borwein and A. S. Lewis, "Partially finite convex programming. I. Quasi relative interiors and duality theory," Mathematical Programming, vol. 57, no. 1, pp. 15-48, 1992.

[10] R. I. Boţ, E. R. Csetnek, and G. Wanka, “Regularity conditions via quasi-relative interior in convex programming," SIAM Journal on Optimization, vol. 19, no. 1, pp. 217-233, 2008.

[11] G. Kassay and K. Nikodem, "Lagrangian multiplier rule for set-valued optimization," Nonlinear Analysis Forum, vol. 6, no. 2, pp. 363-369, 2001.

[12] Y. W. Huang, "Generalized cone-subconvexlike set-valued maps and applications to vector optimization," Journal of Chongqing University, vol. 1, no. 2, pp. 67-71, 2002.

[13] Y. D. Hu and Z. Q. Meng, Convex Analysis and Nonsmooth Analysis, Shanghai Science \& Technology Press, Shanghai, China, 2000.

[14] J. van Tiel, Convex Analysis, John Wiley \& Sons, New York, NY, USA, 1984.

[15] S. Z. Shi, Convex Analysis, Shanghai Science \& Technology Press, Shanghai, China, 1990.

[16] Z. F. Li, "Benson proper efficiency in the vector optimization of set-valued maps," Journal of Optimization Theory and Applications, vol. 98, no. 3, pp. 623-649, 1998.

[17] G. Y. Chen and W. D. Rong, "Characterizations of the Benson proper efficiency for nonconvex vector optimization," Journal of Optimization Theory and Applications, vol. 98, no. 2, pp. 365-384, 1998.

[18] J. Borwein, "Proper efficient points for maximizations with respect to cones," SIAM Journal on Control and Optimization, vol. 15, no. 1, pp. 57-63, 1977.

[19] Y. H. Xu and S. Y. Liu, "Benson proper efficiency in the nearly cone-subconvexlike vector optimization with set-valued functions," Applied Mathematics-A Journal of Chinese Universities Series B, vol. 18, no. 1, pp. 95-102, 2003. 